\title{
A Task Learning Mechanism for the Telerobots
}

\author{
Jing Luo \\ Key Laboratory of Autonomous Systems and Networked Control, \\ College of Automation Science and Engineering, \\ South China University of Technology, \\ Guangzhou 510640, P. R. China \\ jingluo.ac@gmail.com \\ Chenguang Yang* \\ Bristol Robotics Laboratory, \\ University of the West of England, Bristol, UK \\ cyang@ieee.org \\ Qiang Li \\ Neuroinformatics Group, CITEC, \\ Bielefeld University, Bielefeld, Germany \\ qli@techfak.uni-bielefeld.de \\ Min Wang \\ Key Laboratory of Autonomous Systems and Networked Control, \\ College of Automation Science and Engineering, \\ South China University of Technology, \\ Guangzhou 510640, P. R. China \\ auwangmin@scut.edu.cn
}

Received 26 June 2018

Accepted 25 March 2019

Published 9 May 2019

Telerobotic systems have attracted growing attention because of their superiority in the dangerous or unknown interaction tasks. It is very challenging to exploit such systems to implement complex tasks in an autonomous way. In this paper, we propose a task learning framework to represent the manipulation skill demonstrated by a remotely controlled robot. Gaussian mixture model is utilized to encode and parametrize the smooth task trajectory according to the observations from the demonstrations. After encoding the demonstrated

${ }^{*}$ Corresponding author.

This is an Open Access article published by World Scientific Publishing Company. It is distributed under the terms of the Creative Commons Attribution 4.0 (CC-BY) License. Further distribution of this work is permitted, provided the original work is properly cited. 


\section{J. Luo et al.}

trajectory, a new task trajectory is generated based on the variability information of the learned model. Experimental results have demonstrated the feasibility of the proposed method.

Keywords: Telerobotic systems; Gaussian mixture model; Gaussian mixture regression; task model; human-robot interaction.

\section{Introduction}

With the development of sciences and technologies, robots have been widely used in the industry, life, education, entertainment, and military domains. ${ }^{1-7}$ Restricted by many factors, e.g., sensor technologies, control technologies, mechanism design, etc., robots cannot achieve full autonomy. In structural environments, such as automobile production line, robots can do amazing job because the whole manipulation process has been preprogrammed. These technologies, however, cannot directly transfer to the robots which are working in the unstructured and uncertainty environment. On this point, the telerobot is a practical choice which can be remotely controlled by an operator. A telerobotic system consists of five parts: a human operator, a master device, a remote slave device, a communication channel, and an interaction environment. A human operator remotely controls a robot to perform a task in dangerous or unknown environments. The telerobotic system integrates the humans' intelligence and robots' superiorities for increasingly complex task scenarios and interaction environments. Combining the advantages of the robot and operator, the telerobots are widely used in telesurgery, deep-sea/space exploration, nuclear processing, etc. domains.

However, because of time delays, model uncertainties and lack of transparencies for the teleoperated system, it is challenging for the operator to control the remote robot in an easy way. Many works have concentrated on the control algorithms to cope with the problems of model uncertainties and time delays. Yang et al. proposed neural networks (NNs) and automatic collision method to deal with the uncertainties of kinematics and dynamics for a Baxter robot. $^{8} \mathrm{~A}$ radial basis function $\mathrm{NNs}$ (RBFNNs) and wave variable method were presented to compensate the influences of time delay and dynamics uncertainties. ${ }^{9}$ The authors developed an adaptive fuzzy control method to deal with the system's uncertainties and time delay for a dualmaster-single-slave teleoperated system. ${ }^{10,11}$ Sun et al. presented a wave-based timedomain passivity method to enhance the transparency of a four-channel teleoperated system. ${ }^{12}$ Besides, other control algorithms, for examples, hybrid position/force control, impedance control, and fuzzy control method and so on, have widely applied in teleoperation to deal with the issues. ${ }^{13-17}$ These advanced control algorithms effectively improve the performance of the telerobotic system. Yang et al. developed a variable controller with tremor attenuation filter which involves muscle activation for the purpose of personalized control in a dynamic environment. ${ }^{18} \mathrm{~A}$ human-in-theloop framework was proposed to adapt the change of human behavior and ensure a optimal teleimpedance in the process of teleoperation. ${ }^{19}$ Generally, human or human upper limb is a main component of the teleoperated system. The authors analyzed 
the cost energy of the upper limb via evaluation of hand grasp pressure and passivity interaction for teleoperation. ${ }^{20}$ Shahbazi et al. presented a passivity method with respect to human upper limb for the purpose of the natural and safe human-robot interaction (HRI). ${ }^{21}$ Potential answers were developed to deal with the influence factors which involve human in terms of frame rates, image issue, and so on. ${ }^{22}$ By introducing the human-centered interaction methods, the performance of the teleoperated system can be strengthened in the process of control.

The above methods are mainly used to deal with the problems of time delays and model uncertainties. However, it is hard to obtain an accurate model in the presence of environment uncertainty and load uncertainty. Alternatively, the algorithm based on machine learning is a worth choice to solve the problem of estimations of robot model in dynamics and kinematics. Robot learning is an alternative solution to estimate the relationship among operator, the robot, and the executed task. Human operators face a huge manipulation pressure and workload in traditional teleoperation. In some extreme cases, operators even need to control every joints of the robot in order to guide it to finely interact with environment. One solution for easing operator's workload is that the robot learns the manipulation skill offline and implements the manipulation task online according to its perception capability. Along this direction, one very important direction is how robot can learn a skill by human demonstration. The authors developed a dynamical movement primitive (DMP) algorithm with sEMG signal to construct the learned skill and stiffness by using the task trajectories and stiffness information. ${ }^{23,24} \mathrm{~A}$ human motion intention recognition approach was proposed to identify the object and grasp configuration by employing hidden Markov model (HMM) in the teleoperated system. ${ }^{25}$ Tanwani et al. proposed a hidden semi-Markov model (HSMM) algorithm to generate a task model for the purpose of assistance of the human operator. ${ }^{26}$ An impedance control with haptic method was proposed for an assembly in order to learn the task through collection of motion and stiffness messages. ${ }^{27}$ HMM and dynamic time warping (DTW) method were used to learn a complex task by using the task trajectories. ${ }^{28}$ The researchers employed HMM and Gaussian mixture model (GMM) to learn a pouring task and a container-emptying task for a bilateral teleoperated system. ${ }^{29,30}$ A small variance asymptotic method which involved online robot learning was developed to learn a task model and achieve human intention recognition. ${ }^{31}$ Based on shared control, Zeestraten et al. proposed a programming by demonstration (PbD) method which was used to learn a task with state sharing and to improve the task performance. $^{32}$

In order to improve the working efficiency of task and achieve automated task generation, this paper develops a robot task trajectory learning based on machine learning. First, a DTW method is implemented to deal with the problems of temporal mismatch for demonstrated observations. The demonstrated observations can be normalized in the same time domain by using DTW method. Second, a GMM method is used to encode the task trajectory for the purposes of description of relationship among the robot, teleoperator, and the task. Finally, a Gaussian mixture 


\section{J. Luo et al.}

regression (GMR) method is employed to generate continuous, smooth, and statebased task trajectory according to the variability of the demonstrations. In this work, the proposed method brings the following benefits: (1) Efficiency: Compared with the traditional direct teleoperation, the proposed method enables the telerobot to perform a task automatically through several demonstrations. This would be great because it does not require human operator for repetitive task. (2) Security: The teleoperated system does not need human operators all the time. Instead, human operator can concentrate on the decision-making works in teleoperation.

The rest of this paper is organized as follows. Sec. 2 shows the proposed methods composed of DTW, GMM, and GMR. Sec. 3 presents the experimental results. Finally, conclusion is draw in Sec. 4, in which future work is also mentioned.

\section{Methodology}

\subsection{The description of task learning}

In this paper, the system includes 3 modules as shown in Fig. 1: Human demonstrations, robot learning and preproduction, and robot execution.

- Human demonstration module: The function of this module is that an operator remotely controls a slave robot to perform tasks via master haptic device. The trajectories including position, velocity of the robot's end-effector are recorded. In this teleoperated system, a operator controls the master via the position of master device. Because of the different kinematics structure between the master device and the slave robot, we are using the following coordinate transformation:

$$
\left[\begin{array}{l}
\mathbf{x} \\
\mathbf{y} \\
\mathbf{z}
\end{array}\right]=\mathbf{O}_{s m} \times\left\{\boldsymbol{\Lambda}\left[\begin{array}{l}
\mathbf{x}_{m} \\
\mathbf{y}_{m} \\
\mathbf{z}_{m}
\end{array}\right]+\mathbf{b}\right\},
$$

where $\mathbf{x}, \mathbf{y}, \mathbf{z}$ and $\mathbf{x}_{m}, \mathbf{y}_{m}, \mathbf{z}_{m}$ are the position vectors of the slave and the master in task space, respectively. $\mathbf{O}_{s m}$ is the rotation transformation matrix, $\boldsymbol{\Lambda}$ is the scale factor to adjust the workspace between the master and the slave. $\mathbf{b}$ is the position correction term in $X / Y / Z$ directions.

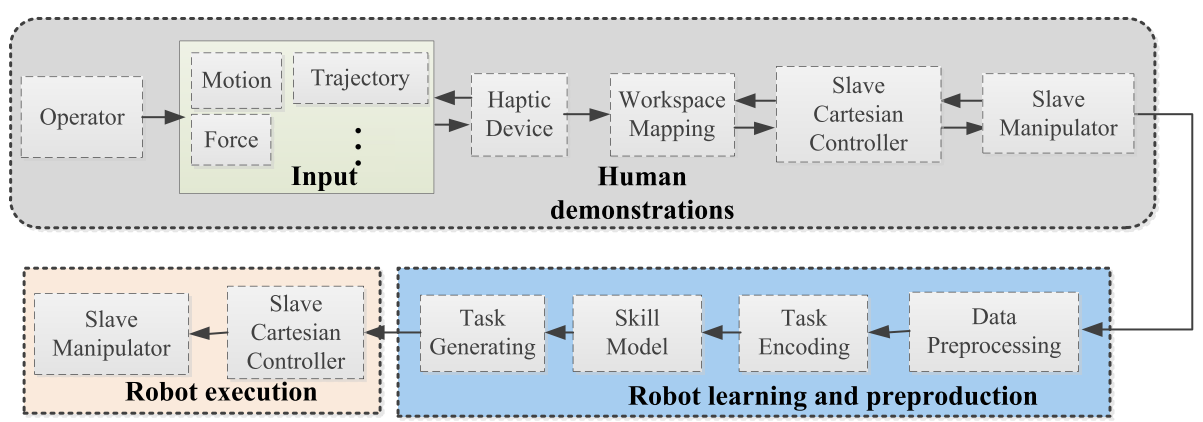

Fig. 1. Flowchart for task demonstration, learning and control. 
- Robot learning and reproduction module: The robot learning module includes data preprocessing, task encoding, and task generating. The function of data preprocessing is to normalize the demonstrated data into a unified time domain. We employ a GMM method based on statistical learning method to encode human skill and develop a GMR method to generate a learned skill model for the slave device according to the new situation.

- Robot execution module: The main function of this module is to enable the robot following the trajectory generated from robot learning module. To this end, the slave robot executes the generalized task learned from the demonstrated robot trajectories.

In order to describe the proposed method more clearly, a procedure of the algorithm and a schematic diagram of the proposed method are outlined in Algorithm 1 and Fig. 2, respectively.

\subsection{Data preprocessing with DTW}

Given the successfully designed human demonstration module, position and velocity of the robot end-effector are sampled from the multiple task demonstrations.

In multiple demonstration, the implementation time of the tasks is not the same. We processed the sample data by employing DTW method to keep data in the same time domain. ${ }^{33}$ DTW algorithm was widely used in speech recognition to deal with the problem of similarity for two different temporal sequences. ${ }^{34,35}$ By introducing the boundary, monotonicity and step size conditions, the similarity can be represented as follows:

$$
\begin{aligned}
D_{\text {opt }}\left\{L\left(x_{1}\right), L\left(x_{2}\right)\right\}= & \left(x_{1}-x_{2}\right)^{2} \\
& + \begin{cases}0 & \text { when } L\left(x_{1}\right)=L\left(x_{2}\right)=1, \\
D\left\{L\left(x_{1}\right), L\left(x_{2}-1\right)\right\} & \text { when } L\left(x_{1}\right)=1, \quad L\left(x_{2}\right)>1, \\
D\left\{L\left(x_{1}-1\right), L\left(x_{2}\right)\right\} & \text { when } L\left(x_{1}\right)>1, \quad L\left(x_{2}\right)=1, \\
\min \left\{D\left\{L\left(x_{1}\right), L\left(x_{2}-1\right)\right\},\right. & \\
D\left\{L\left(x_{1}-1\right), L\left(x_{2}\right)\right\}, & \\
\left.D\left\{L\left(x_{1}\right), L\left(x_{2}\right)\right\}\right\} & \text { otherwise }\end{cases}
\end{aligned}
$$

where $x_{1}$ and $x_{2}$ are the two different length time trajectories (position and velocity). $L\left(x_{1}\right)$ and $L\left(x_{2}\right)$ are the lengths of two trajectories. $D\left\{L\left(x_{1}\right), L\left(x_{2}\right)\right\}$ and $D_{\text {opt }}\left\{L\left(x_{1}\right)\right.$, $\left.L\left(x_{2}\right)\right\}$ are the wrap path distance and optimal wrap path distance, respectively.

\subsection{Task encoded by GMM}

Reinforcement learning (RL) and HMM have been widely used for effectively encoding the task trajectory. ${ }^{30,36}$ Nevertheless, due to the need of good timeliness for encoding a task, the RL method is limited because of its search space being too large for a relatively complex task. Besides, the process of task encoding is a continuous 
Algorithm 1 Procedure of the proposed method

1. Human remotely control slave arm implement task, the trajectories of end-effector are recorded

$$
X=\left\{X^{i}\right\}_{i=1}^{N}
$$

$X$ : demonstrated trajectories composed by position and velocity.

2. Normalize the demonstrated trajectories

$$
X \leftarrow D_{\text {opt }}\left(x_{1}, x_{2}\right)
$$

$x_{1}, x_{2}$ : Two different lengths time trajectories.

$D_{\text {opt }}()$ : DTW algorithm.

3. Encode the training trajectory

$$
\Theta \leftarrow \text { EM algorithm }
$$

$\Theta$ : parameters of GMM are optimized by EM algorithm.

4. Generate the trajectory for a new test task based on GMM

$$
\begin{gathered}
\text { Compute } \hat{X}_{o}(j), \hat{\sigma}_{o}(j) \text { for } j=1, \ldots, K, \\
\hat{\dot{x}}=\sum_{j=1}^{K} h^{j}\left\{\left[\begin{array}{ll}
A^{j} & b^{j}
\end{array}\right]\left[\begin{array}{ll}
x & 1
\end{array}\right]^{T}\right\}, \\
\hat{x}=\left.x\right|_{t-1}+\sum_{j=1}^{K} h^{j}\left\{\left[\begin{array}{ll}
A^{j} & b^{j}
\end{array}\right]\left[\begin{array}{ll}
x & 1
\end{array}\right]^{T} .\right.
\end{gathered}
$$

$\hat{X}_{o}(j)$ : mean of the output in $j$ th step.

$\hat{\sigma}_{o}$ : variance of the output in $j$ th step.

$A^{j}, b^{j}$ and $h^{j}$ can be computed by (16)-(18).

5. Following the generated trajectory to implement new task

one, while the HMM method is needed to be interpolated based on the discrete sets. Therefore, We propose the combined GMM and GMR method which can cope with the above-mentioned shortcomings in robot learning because of its appropriate search space and continuity.

After the preprocessing, we obtained $N$ normalized, demonstrated sample sequence which can be represented as

$$
X=\left\{X^{i}\right\}_{i=1}^{N}=\left\{x_{t}^{i}, \dot{x}_{t}^{i}\right\}_{t=0, i=1}^{T, N},
$$

where $X \in \mathbb{R}^{M}$ consists of positions and velocities of the slave end-effector. $x_{t} \in \mathbb{R}^{M 1}$ and $\dot{x}_{t} \in \mathbb{R}^{M 2}$ are the position and velocity of the slave. $M=6$ with $M 1=3$-dimensional position and $M 2=3$-dimensional with respect to the observations. 


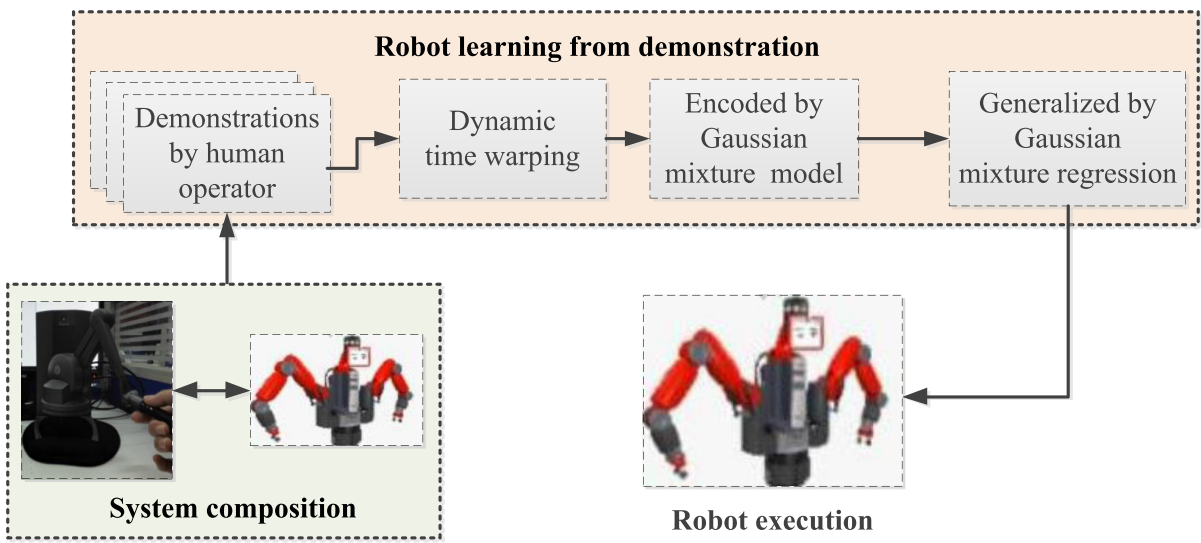

Fig. 2. Schematic diagram of the proposed method.

GMM is developed to encode the demonstrated task from the observation sequences. ${ }^{37,38}$ The probability density function (PDF) $p\left(X^{i}\right)$ can be presented as

$$
p\left(X^{i}\right)=\sum_{j=1}^{K} p(j) \mathcal{N}\left(X^{i} \mid \mu^{j}, \sigma^{j}\right),
$$

where $\mu^{j}$ and $\sigma^{j}$ are the mean values and variances for the observations. $p(j)$ is the prior information for the $K$ Gaussian components in $j$ th step and it satisfies

$$
\sum_{j=1}^{K} p(j)=\sum_{j=1}^{K} \pi(j)=1
$$

$N\left(X^{i} \mid \mu^{j}, \sigma^{j}\right)$ is the Gaussian distribution of the observations given as

$$
N\left(X^{i} \mid \mu^{j}, \sigma^{j}\right)=\frac{1}{\sqrt{(2 \pi)^{3}\left|\sigma^{j}\right|}} \exp \left\{-\frac{\left(X^{i}-\mu^{j}\right)^{T} \sigma^{j-1}\left(X^{i}-\mu^{j}\right)}{2}\right\} .
$$

Inspired by Ref. 39, (4) based on (5)-(6) for the demonstrated observations can be rewritten as

$$
\begin{aligned}
N\left(x_{t}^{i}, \dot{x}_{t}^{i} \mid \Theta\right)= & p\left(X^{i}\right) \\
= & \sum_{j=1}^{K} p(j) \mathcal{N}\left(X^{i} \mid \mu^{j}, \sigma^{j}\right) \\
= & \sum_{j=1}^{K} \pi(j) N\left(x_{t}^{i}, \dot{x}_{t}^{i} \mid \mu^{j}, \sigma^{j}\right) \\
= & \sum_{j=1}^{K} \pi(j) \frac{1}{\sqrt{(2 \pi)^{3}\left|\sigma^{j}\right|}} \\
& \times \exp \left\{-\frac{\left(X^{i}-\mu^{j}\right)^{T} \sigma^{j-1}\left(X^{i}-\mu^{j}\right)}{2}\right\},
\end{aligned}
$$




\section{J. Luo et al.}

where $\Theta=\left\{\Theta^{j}\right\}_{j=1}^{K}=\left\{\pi^{1}, \mu^{1}, \sigma^{1} ; \ldots ; \pi^{j}, \mu^{j}, \sigma^{j}, \ldots, \pi^{K}, \mu^{K}, \sigma^{K}\right\}$ are the parameters of Gaussian component. In this paper, the demonstrated observations are regarded as independent Gaussian distribution. According to the demonstrated observations, the parameters of GMM can be estimated by employed expectation-maximization (EM) method. The values of EM method are initialized by using $k$-means clustering algorithm.

\subsection{Task generated by GMR}

According to the demonstrated observation sequence, GMR method is employed to generate a generalized task model after encoded by GMM. ${ }^{40-42}$ By employing the joint probability distribution, the observation sequence, means matrix, and covariance matrix can be described as

$$
\begin{gathered}
\mu^{j}=\left[\begin{array}{l}
\mu_{i}^{j} \\
\mu_{o}^{j}
\end{array}\right]=\left[\begin{array}{l}
\mu_{x}^{j} \\
\mu_{\dot{x}}^{j}
\end{array}\right], \\
\sigma_{j}=\left[\begin{array}{cc}
\sigma_{i}^{j} & \sigma_{i o}^{j} \\
\sigma_{o i}^{j} & \sigma_{o}^{j}
\end{array}\right]=\left[\begin{array}{cc}
\sigma_{x x}^{j} & \sigma_{x \dot{x}}^{j} \\
\sigma_{\dot{x} x}^{j} & \sigma_{\dot{x} \dot{x}}^{j}
\end{array}\right] .
\end{gathered}
$$

The estimation values of conditional distribution for output data $\hat{X}_{o}^{j}$ can be computed according to the conditional output $X_{o}^{j}$ and given $X_{i}$ as follows:

$$
\begin{gathered}
\hat{X}_{o}^{j}=\mu_{o}^{j}+\sigma_{o i}^{j}\left(\sigma_{i}^{j}\right)^{-1}\left(X_{i}-\mu_{i}^{j}\right) \\
=\mu_{\dot{x}}^{j}+\sigma_{\dot{x} x}^{j}\left(\sigma_{x x}^{j}\right)^{-1}\left(X_{i}-\mu_{x}^{j}\right), \\
\hat{\sigma}_{o}^{j}=\sigma_{o}^{j}-\sigma_{o i}^{j}\left(\sigma_{i}^{j}\right)^{-1} \sigma_{i o}^{j} \\
=\sigma_{\dot{x}}^{j}-\sigma_{\dot{x} x}^{j}\left(\sigma_{x x}^{j}\right)^{-1} \sigma_{x \dot{x}}^{j} .
\end{gathered}
$$

Motivated by the related works in Refs. 43 and 44, the conditional distribution of output data $\hat{X}_{o}$ for $K$ Gaussian component as

$$
\begin{aligned}
& \hat{X}_{o}=\sum_{j=1}^{K} \beta^{j} \hat{X}_{o}^{j} \\
&=\sum_{j=1}^{K} \beta^{j}\left\{\mu_{\dot{x}}^{j}+\sigma_{\dot{x} x}^{j}\left(\sigma_{x x}^{j}\right)^{-1}\left(X_{i}-\mu_{x}^{j}\right)\right\} \\
& \beta^{j}=\frac{p\left(X_{i} \mid j\right)}{\sum_{j=1}^{K} p\left(X_{i} \mid j\right)} \\
&=\frac{p(j) p\left(X_{i} \mid j\right)}{\sum_{j=1}^{K} p(j) p\left(X_{i} \mid j\right)}
\end{aligned}
$$




$$
\begin{aligned}
\hat{\sigma}_{o} & =\sum_{j=1}^{K} \beta^{j 2} \hat{\sigma}_{o}^{j} \\
& =\sum_{j=1}^{K} \beta^{j 2}\left\{\sigma_{\dot{x}}^{j}-\sigma_{\dot{x} x}^{j}\left(\sigma_{x x}^{j}\right)^{-1} \sigma_{x \dot{x}}^{j}\right\} .
\end{aligned}
$$

Thus, a generated task model is obtained based on GMR. The generated motion from the learned model can perform smoothly without paying attention to the inverse kinematics problem of the slave. This would greatly improve the real-time ability of the telerobotic systems for the automated task.

According to the conditional probability of GMR and a current given position $x$, we can obtain the desired velocity as

$$
\hat{\dot{x}}=\sum_{j=1}^{K} h^{j}\left\{\left[\begin{array}{ll}
A^{j} & b^{j}
\end{array}\right]\left[\begin{array}{ll}
x & 1
\end{array}\right]^{T}\right\},
$$

where

$$
\begin{gathered}
A^{j}=\sigma_{\dot{x} x}^{j}\left(\sigma_{x x}^{j}\right)^{-1}, \\
b^{j}=\hat{\mu}_{\dot{x}}^{j}-\sigma_{\dot{x} x}^{j}\left(\sigma_{x x}^{j}\right)^{-1} \mu_{x}^{j}, \\
h^{j}=\frac{\mathcal{N}\left(x \mid \mu_{x}^{j}, \sigma_{x x}^{j}\right)}{\sum_{j=1}^{K} \mathcal{N}\left(x \mid \mu_{x}^{j}, \sigma_{x x}^{j}\right)},
\end{gathered}
$$

where $j=1, \ldots, K$ in $(16)-(18)$.

According to Euler integration, the desired position in Cartesian space at time $t$ is updated based on the computed desired velocity $\hat{\dot{x}}$ as follows:

$$
\hat{x}=\left.x\right|_{t-1}+\sum_{j=1}^{K} h^{j}\left\{\left[\begin{array}{ll}
A^{j} & b^{j}
\end{array}\right]\left[\begin{array}{ll}
x & 1
\end{array}\right]^{T}\right\} .
$$

\section{Results and Discussion}

\subsection{Experimental setup}

We demonstrate our proposed method using a telerobotic system shown in Fig. 3, which is composed of Touch X (haptic device) and a Baxter robot. Touch X communicates with Baxter robot via a User Datagram Protocol (UDP) module. A cleaning task is performed to validate the effectiveness of the proposed method with different initial conditions based on GMM and GMR methods. In the cleaning task, a gray cardboard and a yellow cube are used as a cleaning tool and a rubbish, respectively. A human operator tele-controls the right arm of Baxter robot to sweep the rubbish into the red garbage bucket. There exist several demonstrations in this task. By learning the cleaning task, the robot can be executed successfully in different initial positions. 


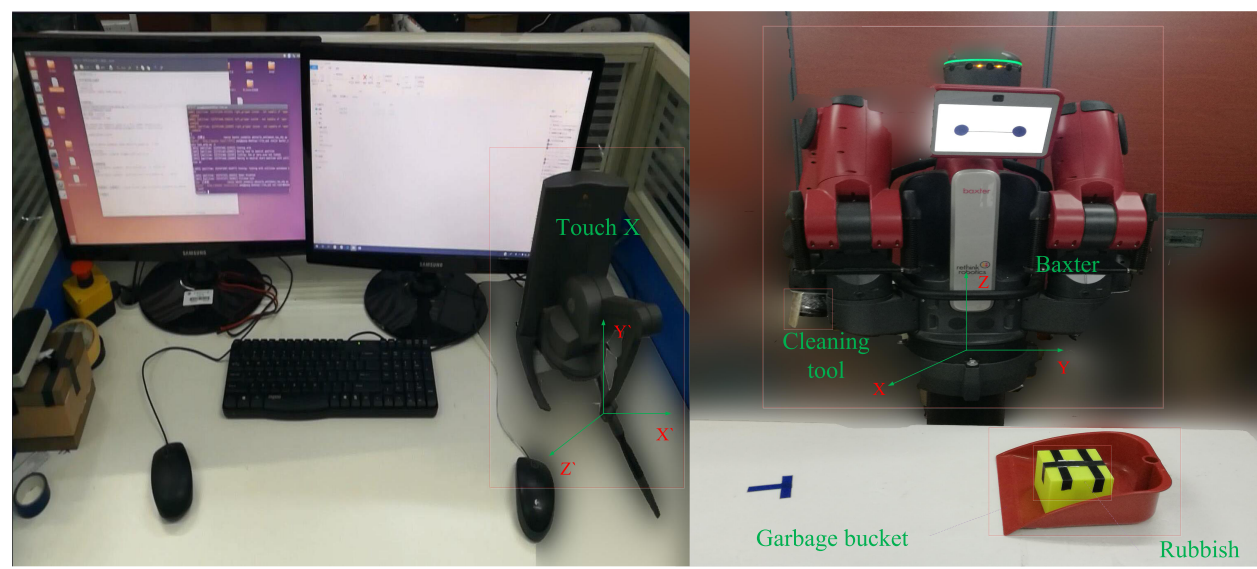

Fig. 3. Experimental setup for a cleaning task.

\subsection{Demonstrated observation preprocessing}

The cleaning task is demonstrated 10 times from different initial places through a teleoperated mode. Figures 4 and 5 show the demonstrated trajectories and the unified trajectories by using DTW method. Figures 4(a)-4(c) and Figs. 5(a)-5(c), respectively, show the slave's end-effector trajectory before and after DTW method is

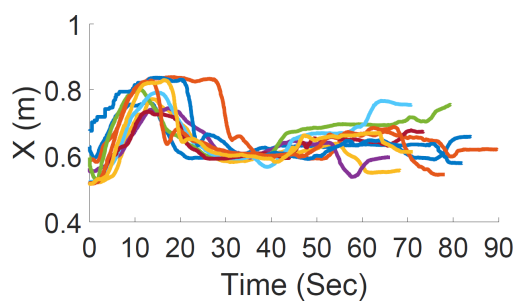

(a) Demonstrated observations in $X$-axis

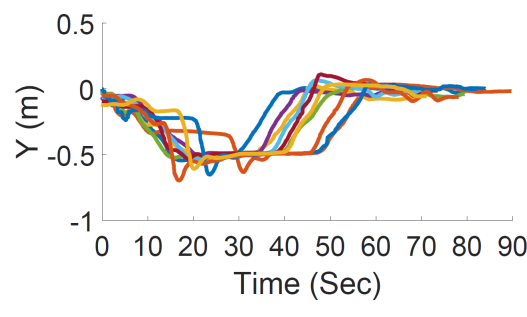

(b) Demonstrated observations in $Y$-axis

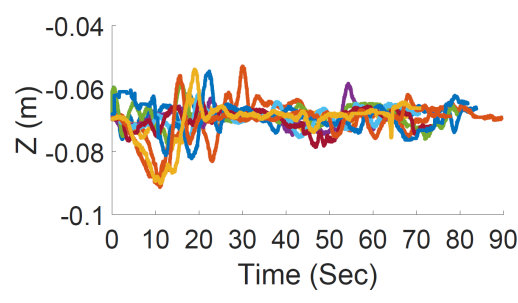

(c) Demonstrated observations in Z-axis

Fig. 4. Left: (a) shows the collection of demonstrated observations without DTW in $X$-axis from 10 demonstrations. Middle: (b) is the collection of demonstrated observations without DTW in $Y$-axis from 10 demonstrations. Right: (c) displays the collection of demonstrated observations without DTW in $Z$-axis from 10 demonstrations. 


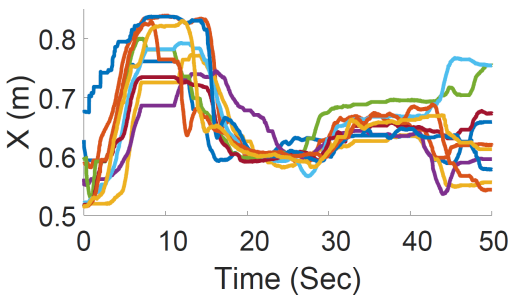

(a) Data preprocessing by using DTW method in $X$-axis

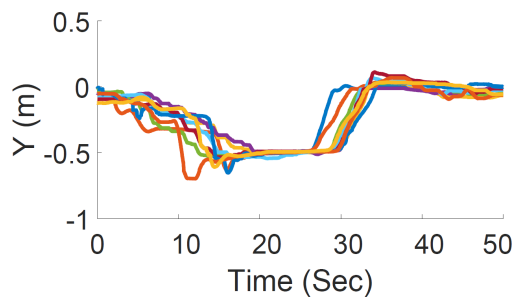

(b) Data preprocessing by using DTW method in $Y$-axis

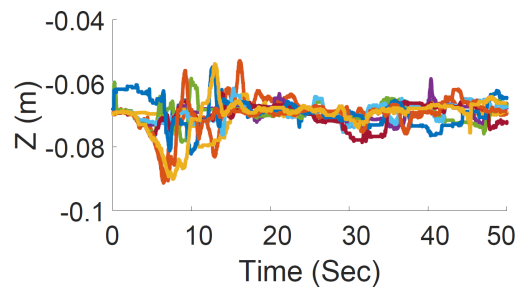

(c) Data preprocessing by using DTW method in Z-axis

Fig. 5. Left: (a) shows the collection of demonstrated observations with DTW in $X$-axis. Middle: (b) is the collection of demonstrated observations with DTW in $Y$-axis. Right: (c) displays the collection of demonstrated observations with DTW in Z-axis.

applied. Figures 4(a)-4(c) display the time spending in each demonstration before using DTW method. It can be concluded that each demonstration takes different amounts of time to complete the cleaning task. The demonstrated trajectories are performed in different initial positions. As shown in Figs. 5(a)-5(c), the trajectories are normalized properly in the same time domain. It can be seen that the trajectories by using DTW method are successful normalizations. The results indicate that the DTW method improves the smoothness of the demonstrated trajectories and ensures the synchronicity of the motion in the same time domain.

\subsection{Results}

As shown in Fig. 6, the demonstrated trajectories are divided into 10 times to indicate the similarity of each demonstrated cleaning task in the same time domain. It can be seen that the curve of each demonstration has a similar shape in comparison with other curves.

In this experiment, the GMM method is employed to encode the cleaning task. From Figs. 7(a)-7(c), it shows that the related GMM model can be encoded by 5 states from 10 demonstrations. ${ }^{a}$ The parameters of GMM model indicate the cleaning task encoding information via 10 unified demonstration trajectories. The information of the task can be known via GMM parameter $\left\{\Theta^{j}\right\}_{j=1}^{K}$. Additionally,

a The number of states are predetermined according to the task. 
J. Luo et al.

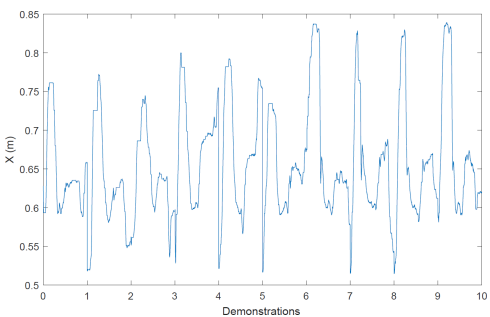

(a) Demonstrations $(N=10)$ in $X$-axis for a cleaning task

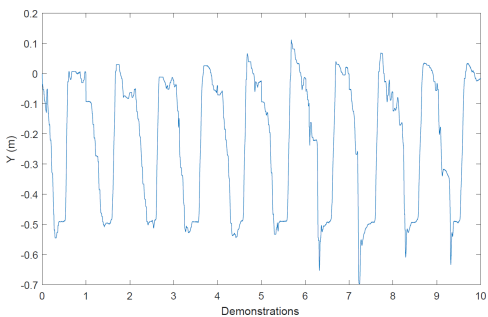

(b) Demonstrations $(N=10)$ in $Y$-axis for a cleaning task

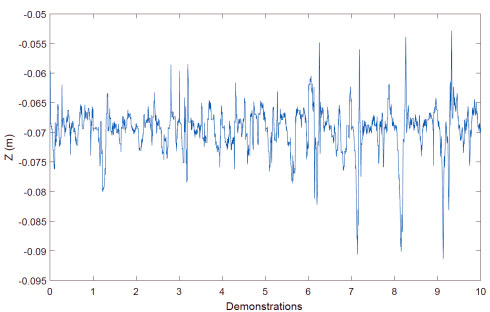

(c) Demonstrations $(N=10)$ in $Z$-axis for a cleaning task

Fig. 6. Left: (a) shows 10 times demonstrations for a cleaning task in $X$-axis. Middle: 10 times demonstrations for a cleaning task in $Y$-axis are shown in (b). Right: 10 times demonstrations for a cleaning task in $Z$-axis is shown in (c).

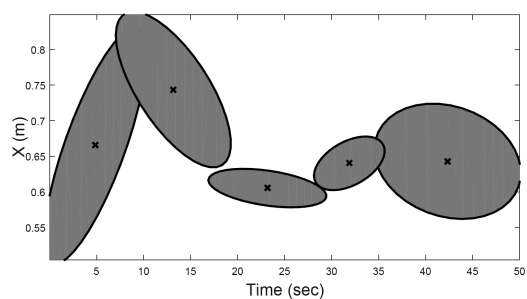

(a) Encoded models by using GMM in $X$-axis

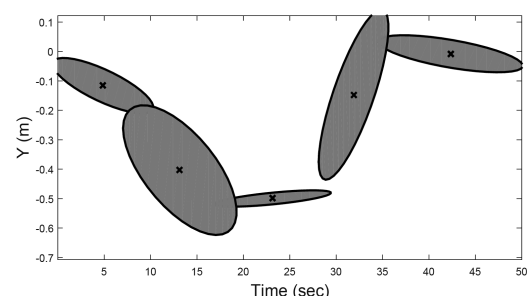

(b) Encoded models by using GMM in $Y$-axis

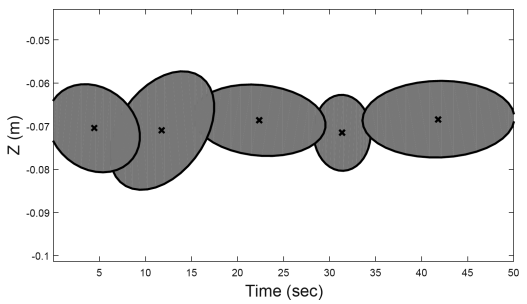

(c) Encoded models by using GMM in $Y$-axis

Fig. 7. Left: (a) shows the task encoding by using GMM in $X$-axis. Middle: (b) is task encoding by using GMM in $Y$-axis. Right: (c) displays the task encoding by using GMM in $Z$-axis. 


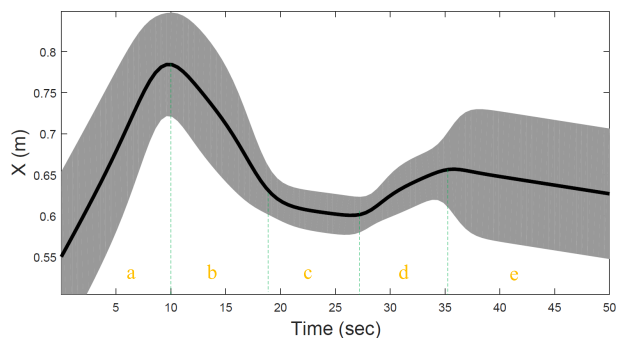

(a) Regressed models by using GMR in $X$-axis

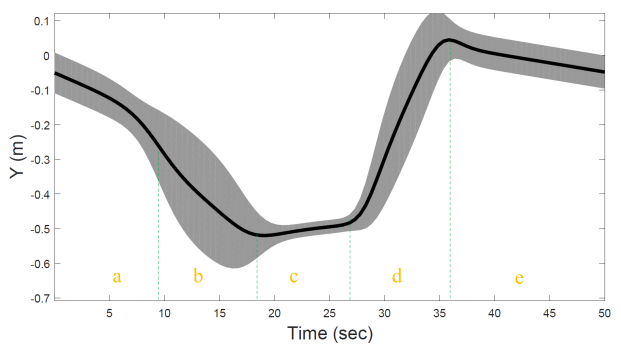

(b) Regressed models by using GMR in $Y$-axis

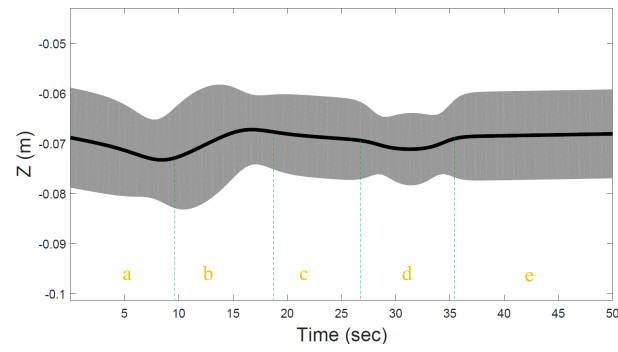

(c) Regressed models by using GMR in Z-axis

Fig. 8. Left: (a) shows the generation task by using GMR in $X$-axis. Middle: (b) is generation task by using GMR in $Y$-axis. Right: (c) displays the generation task by using GMR in $Z$-axis.

the cleaning motion varies mainly in the $X$ and $Y$-axes, while it is relatively stable in $Z$-axis. The encoding task indicates that the cleaning task has great variability in $X-Y$ space.

In the generating phase, the desired position can be obtained according to GMR method. The results of generating task are plotted in Figs. 8(a)-8(c) based on the learned model. There is a smooth generated trajectory in $X / Y / Z$-axes according to (10)-(14). It is noted that the generated trajectories could be adjusted

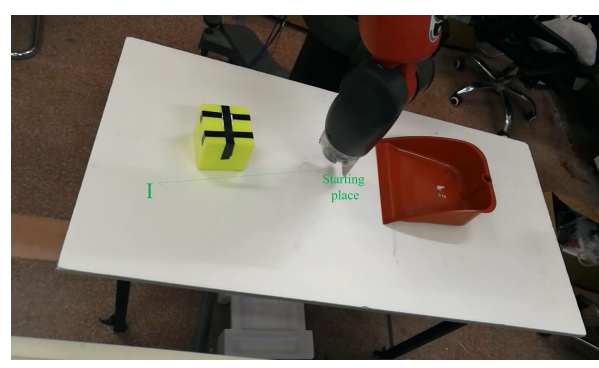

(a) Robot execution process from starting place to phase I for a cleaning task

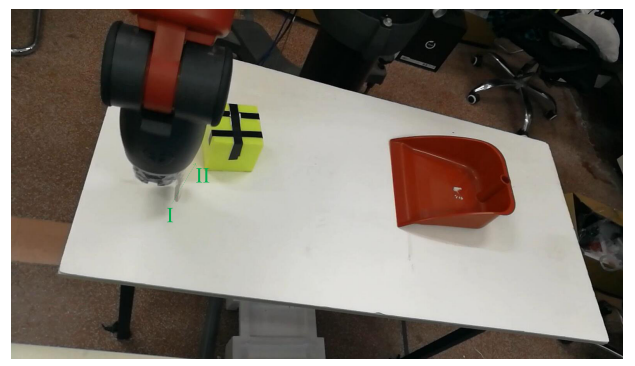

(b) Robot execution process from phase I to phase II for a cleaning task

Fig. 9. The robot execution for a cleaning task from phase I to phase V. 


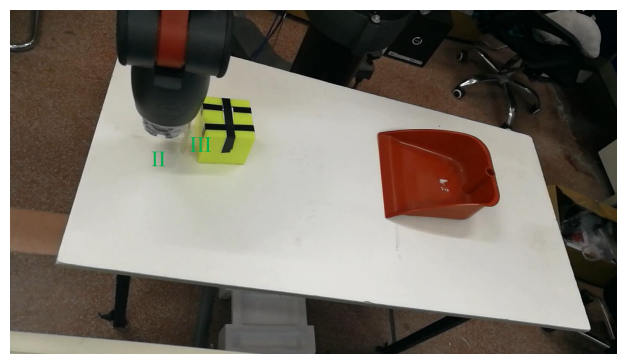

(c) Robot execution process from phase II to phase III for a cleaning task

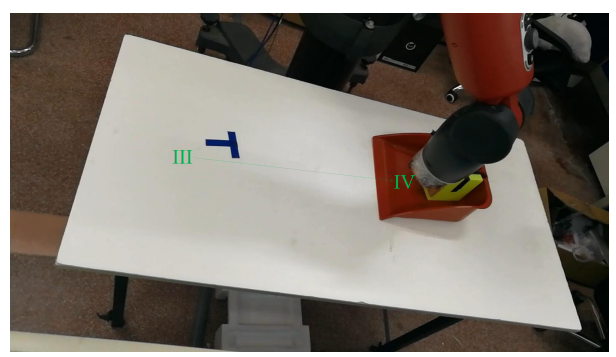

(e) Robot execution process from phase III to phase IV for a cleaning task

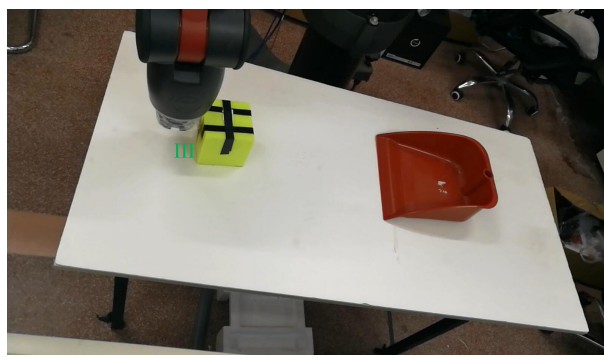

(d) Robot execution process in phase III pausing for a moment for a cleaning task

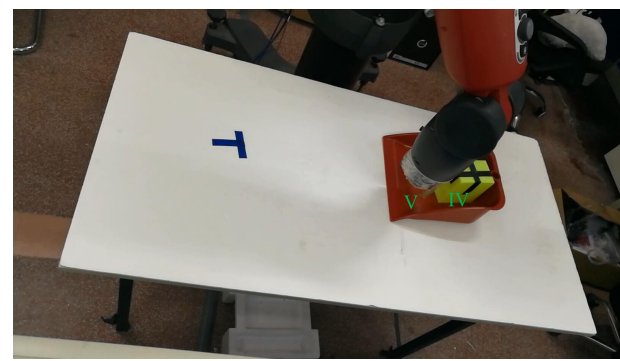

(f) Robot execution process from phase IV to phase $\mathrm{V}$ for a cleaning task

Fig. 9. (Continued)

according to the feedback controller of the slave from different initial position (Figs. 8(a)-8(c)).

The robot execution process of the cleaning task is presented in Fig. 9. In Fig. 9(a), the robot is performed from a given initial position which is different from the trainings. In order to describe the execution process of the slave, we artificially divide the process into five phases (phases I-V). In phase I, the motion process can be updated according to (15)-(18) via a given initial position. This phase is corresponded with the phase $a$ in generating process. Accordingly, Figs. 9(b)-9(f) indicate the rest of the cleaning task performed process (four phases) corresponding to the another phases (b)-(f) which are generated by using GMR method, respectively. In the robot execution process, the cleaning task is successfully implemented by using GMM-GMR method.

\section{Conclusion and Future Work}

This paper proposes a task learning framework for the teleoperated robot to explore the relationship among the robot, operator, and the task. In this paper, we adopt to use a DTW method which is used to normalize the 10 demonstrated observations 
with different time scale. We propose a new learning framework which employs GMM method to encode the demonstrated trajectory of robot end-effector while a operator remotely controls the robot implementing a cleaning task. In the framework, we use GMR to generalize the training result to new situation (different initial position), in our case, in the evaluation experiment, the cleaning task starts from a new position. Experimental results indicate that the proposed learning framework is feasible. After 10 times demonstrations, the proposed method successfully generates a trajectory which has different initial positions with the training set. Combining with the robot cartesian controller, the clean task in the new situation is implemented correctly. In the future, impedance and force information of end-effector of the robot involving human-robot interaction should be considered. The time-delay issue of the teleoperation system should also be noted. ${ }^{45,46}$ Furthermore, more industrial task scenarios should be explored in practical applications.

\section{Acknowledgments}

This work was partially supported by National Nature Science Foundation (NSFC) under Grants 61861136009 and 61811530281, by Engineering and Physical Sciences Research Council (EPSRC) under Grant EP/S001913, and by Changzhou International Collaboration Grant CZ20170018.

\section{References}

1. U. A. Hofmann, T. Btzer, O. Lambercy and R. Gassert, Design and evaluation of a bowden-cable-based remote actuation system for wearable robotics, IEEE Robot. Autom. Lett. 3(3) (2018) 2101-2108.

2. M. T. Pope, C. W. Kimes, H. Jiang, E. W. Hawkes, M. A. Estrada, C. F. Kerst et al., A multimodal robot for perching and climbing on vertical outdoor surfaces, IEEE Trans. Robot. 33(1) (2017) 38-48.

3. L. P. E. Toh, A. Causo, P. W. Tzuo, I. Chen and S. H. Yeo, A review on the use of robots in education and young children, J. Educ. Technol. Soc. 19(2) (2016) 148.

4. N. Reich-Stiebert and F. Eyssel, Learning with educational companion robots? Toward attitudes on education robots, predictors of attitudes, and application potentials for education robots, Int. J. Soc. Robot. 7(5) (2015) 875-888.

5. H. S. Ahn, C. Datta, I. H. Kuo, R. Stafford, N. Kerse, K. Peri et al., Entertainment services of a healthcare robot system for older people in private and public spaces, in IEEE Int. Conf. Automation, Robotics and Applications (ICARA) (IEEE, New York, 2015), pp. 217-222.

6. F. Jentsch, Human-Robot Interactions in Future Military Operations (CRC Press, Boca Ratan, 2016).

7. Q. Wu, Y. Liu and C. Wu, An overview of current situations of robot industry development, in ITM Web of Conf., Vol. 17 (France, 2018), p. 03019.

8. C. Yang, X. Wang, L. Cheng and H. Ma, Neural-learning-based telerobot control with guaranteed performance, IEEE Trans. Cybern. 47(10) (2017) 3148-3159.

9. C. Yang, X. Wang, Z. Li, Y. Li and C. Y. Su, Teleoperation control based on combination of wave variable and neural networks, IEEE Trans. Syst. Man Cybern. Syst. 47(8) (2017) $2125-2136$. 
10. Z. Li, L. Ding, H. Gao, G. Duan and C. Y. Su, Trilateral teleoperation of adaptive fuzzy force/motion control for nonlinear teleoperators with communication random delays, IEEE Trans. Fuzzy Syst. 21(4) (2013) 610-624.

11. Z. Li, Y. Xia, D. Wang, D. H. Zhai, C. Y. Su and X. Zhao, Neural network-based control of networked trilateral teleoperation with geometrically unknown constraints, IEEE Trans. Cybern. 46(5) (2016) 1051-1064.

12. D. Sun, F. Naghdy and H. Du, Wave-variable-based passivity control of four-channel nonlinear bilateral teleoperation system under time delays, IEEE/ASME Trans. Mechatron. 21(1) (2016) 238-253.

13. D. Heck, A. Saccon, N. Van de Wouw and H. Nijmeijer, Guaranteeing stable tracking of hybrid positionforce trajectories for a robot manipulator interacting with a stiff environment, Automatica 63 (2016) 235-247.

14. M. K. Soltani, S. Khanmohammadi, F. Ghalichi and F. Janabi-Sharifi, A soft robotics nonlinear hybrid position/force control for tendon driven catheters, Int. J. Control Autom. Syst. 15(1) (2017) 54-63.

15. Z. Chen, Y. J. Pan and J. Gu, Integrated adaptive robust control for multilateral teleoperation systems under arbitrary time delays, Int. J. Robust Nonlinear Control 26(12) (2016) 2708-2728.

16. D. H. Zhai and Y. Xia, Adaptive fuzzy control of multilateral asymmetric teleoperation for coordinated multiple mobile manipulators, IEEE Trans. Fuzzy Syst. 24(1) (2016) $57-70$.

17. C. Hua, Y. Yang and P. X. Liu, Output-feedback adaptive control of networked teleoperation system with time-varying delay and bounded inputs, IEEE/ASME Trans. Mechatron. 20(5) (2015) 2009-2020.

18. C. Yang, J. Luo, Y. Pan, Z. Liu and C. Y. Su, Personalized variable gain control with tremor attenuation for robot teleoperation, IEEE Trans. Syst. Man Cybern. Syst. 48(10) (2018) 1759-1770.

19. L. Peternel, N. Tsagarakis and A. Ajoudani, A human-robot co-manipulation approach based on human sensorimotor information, IEEE Trans. Neural Syst. Rehabil. Eng. 25(7) (2017) 811-822.

20. S. F. Atashzar, M. Shahbazi, M. Tavakoli and R. V. Patel, A grasp-based passivity signature for haptics-enabled human-robot interaction: Application to design of a new safety mechanism for robotic rehabilitation, Int. J. Robot. Res. 36(5-7) (2017) 778-799.

21. M. Shahbazi, S. F. Atashzar, M. Tavakoli and R. V. Patel, Position-force domain passivity of the human arm in telerobotic systems, IEEE/ASME Trans. Mechatron. 23(2) (2018) 552-562.

22. J. Y. Chen and M. J. Barnes, Human-agent teaming for multirobot control: A review of human factors issues, IEEE Trans. Hum.-Mach. Syst. 44(1) (2014) 13-29.

23. C. Yang, C. Zeng, C. Fang, W. He and Z. Li, A DMPs-based framework for robot learning and generalization of humanlike variable impedance skills, IEEE/ASME Trans. Mechatron. 23(3) (2018) 1193-1203.

24. Y. Chenguang, L. Jing, L. Chao, Li. Miao and D. Shi-Lu, Haptics electromyogrphy perception and learning enhanced intelligence for teleoperated robot, IEEE Trans. Automation Sci. Eng. (2018) 1-10. DOI: 10.1109/TASE.2018.2874454.

25. K. Khokar, R. Alqasemi, S. Sarkar, K. Reed and R. Dubey, A novel telerobotic method for human-in-the-loop assisted grasping based on intention recognition, in 2014 IEEE Int. Conf. Robotics and Automa. (ICRA) (IEEE, New York, 2014), pp. 4762-4769.

26. A. K. Tanwani and S. Calinon, A generative model for intention recognition and manipulation assistance in teleoperation, in 2017 IEEE/RSJ Int. Conf. Intelligent Robots and Systems (IROS) (IEEE, New York, 2017), pp. 43-50. 
27. L. Peternel, T. Petri and J. Babi, Human-in-the-loop approach for teaching robot assembly tasks using impedance control interface, in 2015 IEEE Int. Conf. Robotics and Automation (ICRA) (IEEE, New York, 2015), pp. 1497-1502.

28. A. Vakanski, I. Mantegh, A. Irish and F. Janabi-Sharifi, Trajectory learning for robot programming by demonstration using hidden Markov model and dynamic time warping, IEEE Trans. Syst. Man Cybern. B, Cybern. 42(4) (2012) 1039-1052.

29. L. Rozo, P. Jimnez and C. Torras, Robot learning from demonstration of force-based tasks with multiple solution trajectories, in 2011 15th Int. Conf. Advanced Robotics (ICAR) (2011), pp. 124-129.

30. L. Rozo, P. Jimnez and C. Torras, Force-based robot learning of pouring skills using parametric hidden markov models, in 2013 9th Workshop IEEE in Robot Motion and Control (RoMoCo) (2013), pp. 227-232.

31. A. K. Tanwani and S. Calinon, Small variance asymptotics for non-parametric online robot learning, arXiv:1610.02468.

32. M. J. Zeestraten, I. Havoutis and S. Calinon, Programming by demonstration for shared control with an application in teleoperation, IEEE Robot. Autom. Lett. 3 (2018) $1848-1855$.

33. E. Sabbaghi, M. Bahrami and S. S. Ghidary, Learning of gestures by imitation using a monocular vision system on a humanoid robot, in 2014 Second RSI/ISM Int. Conf. Robotics and Mechatronics (ICRoM) (IEEE, Tehran, Iran, 2014), pp. 588-594.

34. J. Aleotti, A. Cionini, L. Fontanili and S. Caselli, Arm gesture recognition and humanoid imitation using functional principal component analysis, in 2013 IEEE/RSJ Int. Conf. Intelligent Robots and Systems (IROS) (IEEE, New York, 2013), pp. 37523758 .

35. H. C. Lin, T. Tang, Y. Fan, Y. Zhao, M. Tomizuka and W. Chen, Robot learning from human demonstration with remote lead hrough teaching, in 2016 European Control Conf. (ECC) (IEEE, Aalborg, Denmark, 2016), pp. 388-394.

36. J. Kober, J. A. Bagnell and J. Peters, Reinforcement learning in robotics: A survey, Int. J. Robot. Res. 32(11) (2013) 1238-1274.

37. L. Rozo, D. Bruno, S. Calinon and D. G. Caldwell, Learning optimal controllers in human-robot cooperative transportation tasks with position and force constraints, in 2015 IEEE/RSJ Int. Conf. Intelligent Robots and Systems (IROS) (IEEE, New York, 2015), pp. 1024-1030.

38. M. Saveriano and D. Lee, Learning motion and impedance behaviors from human demonstrations, in 2014 11th Int. Conf. Ubiquitous Robots and Ambient Intelligence (URAI) (IEEE, Kuala Lumpur, Malaysia, 2014), pp. 368-373.

39. S. Calinon, E. L. Sauser, A. G. Billard and D. G. Caldwell, Evaluation of a probabilistic approach to learn and reproduce gestures by imitation, in 2010 IEEE Int. Conf. Robotics and Automation (ICRA) (IEEE, New York, 2010), pp. 2671-2676.

40. F. Abi-Farraj, T. Osa, N. P. J. Peters, G. Neumann and P. R. Giordano, A learning-based shared control architecture for interactive task execution, in 2017 IEEE Int. Conf. Robotics and Automation (ICRA) (IEEE, New York, 2017), pp. 329-335.

41. S. Calinon, P. Evrard, E. Gribovskaya, A. Billard and A. Kheddar, Learning collaborative manipulation tasks by demonstration using a haptic interface, in 2009 IEEE Int. Conf. Advanced Robotics (ICAR) (IEEE, New York, 2009), pp. 1-6.

42. M. Power, H. Rafii-Tari, C. Bergeles, V. Vitiello and G. Z. Yang, A cooperative control framework for haptic guidance of bimanual surgical tasks based on learning from demonstration, in 2015 IEEE Int. Conf. Robotics and Automation (ICRA) (IEEE, New York, 2015), pp. 5330-5337. 
43. M. Racca, J. Pajarinen, A. Montebelli and V. Kyrki, Learning in-contact control strategies from demonstration, in 2016 IEEE/RSJ Int. Conf. Intelligent Robots and Systems (IROS) (IEEE, New York, 2016), pp. 688-695.

44. S. Calinon, D. Bruno, M. S. Malekzadeh, T. Nanayakkara and D. G. Caldwell, Humanrobot skills transfer interfaces for a flexible surgical robot, Comput. Methods Programs Biomed. 116(2) (2014) 81-96.

45. A. Yamaguchi, C. G. Atkeson and T. Ogasawara, Pouring skills with planning and learning modeled from human demonstrations, Int. J. Humanoid Robot. 12(3) (2015) 1550030 .

46. L. Jing, Y. Chenguang, W. Ning and W. Min, Enhanced teleoperation performance using hybrid control and virtual fixture, Int. J. Syst. Sci. 50(3) (2019) 451-462.

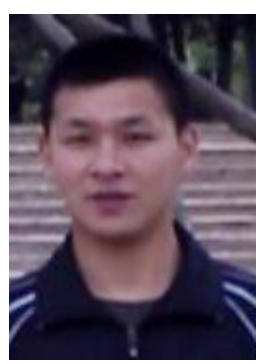

Jing Luo is currently pursuing his Ph.D. degree with the South China University of Technology (SCUT), Guangzhou, China. He was a vising researcher in Laboratoire d'Informatique, de Robotique et de Microélectronique de Montpellier (LIRMM) in 2018. His research interests include robotics, teleoperation, haptics, nonlinear control theory, machine learning, and humanrobot interaction.

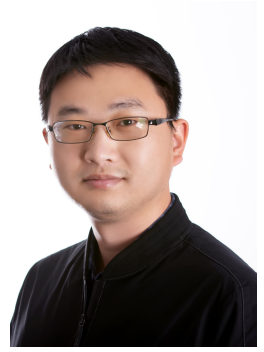

Chenguang Yang is a Professor of Robotics. He received his Ph. D. degree in Control Engineering from the National University of Singapore, Singapore, in 2010 and performed postdoctoral research in human robotics at Imperial College London, London, UK from 2009 to 2010. He has been awarded EU Marie Curie International Incoming Fellowship, UK EPSRC UKRI Innovation Fellowship, and the Best Paper Award of the IEEE Transactions on Robotics as well as over 10 conference Best Paper Awards. His research interest lies in human robot interaction and intelligent system design.

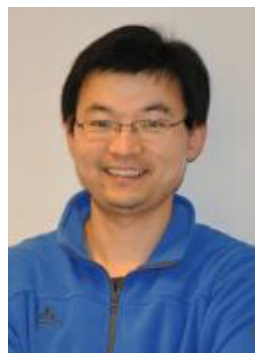

Qiang Li received his Ph.D. in Pattern Recognition and Intelligence Systems from Shenyang Institute of Automation (SIA), Chinese Academy of Sciences (CAS) in 2010. In October, 2009, he was awarded the Honda Research Institute stipend and started his postdoctoral researching at CoR-Lab of Bielefeld University. Currently, he is a Scientific Researcher in neuroinformatics group, CITEC, Bielefeld University. His research interests include robotic dexterous manipulation, tactile sensing, perception and manipulation, multi-modal-based servo control, robotic body schema learning. 


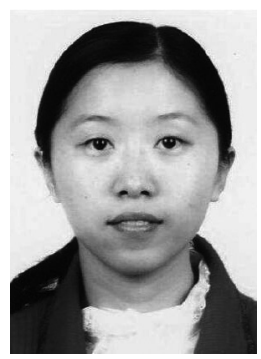

Min Wang received her B.Sc. and M.Sc. degrees from the Department of Mathematics, Bohai University, Jinzhou, China, in 2003 and 2006, respectively; Ph.D. degree from the Institute of Complexity Science, Qingdao University, Qingdao, China, in 2009. From November 2017 to 2018, she is an Academic Visitor in the Department of Information Systems and Computing, Brunel University London, Uxbridge, UK She is currently an Associate Professor with the School of Automation Science and Engineering, South China University of Technology, Guangzhou, China. She has authored or co-authored nearly 30 papers in top international journals. Dr. Wang was a recipient of the Excellent Doctoral Dissertations Award of Shandong Province in 2010, the Science and Technology New Star of Zhujiang, Guangzhou, in 2014, and the youth talent of Guangdong Tezhi Plan in 2016. Her current research interests include nonlinear systems, intelligent control, robot control, and dynamic learning. She is a very active reviewer for many international journals. 